\title{
AVALIAÇÃO DA DEPENDÊNCIA À PRÁTICA DE KARATÊ E A SUA RELAÇÃO COM O TEMPO DE TREINO
}

\author{
ASSESSMENT OF PHYSICAL EXERCISE ADDICTION IN KARATE AND ITS RELATION \\ TO TIME OF ENGAGEMENT
}

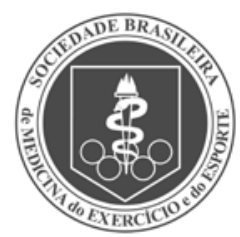

Artigo Original

\section{LA DEPENDENCIA DE LA EVALUACIÓN A LA PRÁCTICA DEL KARATE Y SU RELACIÓN CON EL TIEMPO DE ENTRENAMIENTO}

Luciana Rossi ${ }^{1,2}$ (Nutricionista) Julio Tirapegui ${ }^{1,2}$ (Bioquímico)

\section{Faculdade de Ciências}

Farmacêuticas da Universidade de São Paulo - Departamento de Alimentos e Nutrição Experimental, São Paulo, SP, Brasil.

2. Programa de Pós-Graduação Interunidade em Nutrição Humana Aplicada (PRONUT) da FSP/FCF/FEA da Universidade de São Paulo, São Paulo, SP, Brasil.

\section{Correspondência:}

Faculdade de Ciências

Farmacêuticas Universidade de São Paulo. Av. Prof. Lineu Prestes, 580,

Bloco 14, 05508-900

São Paulo, SP, Brasil.

Irossi@usp.br

\section{RESUMO}

Introdução: a prática de artes marciais tem se tornado uma atividade em grande ascensão, seja no âmbito recreacional ou competitivo. Embora sejam inegáveis os benefícios da prática regular de exercícios físicos, crescem as evidências do excesso de treino entre atletas e desportistas de diferentes modalidades, chegando mesmo à dependência da prática em situações negativas e patológicas. Este excesso, denominado Dependência da Prática de Exercícios (DPE), pode se apresentar na forma primária ou secundária. Objetivo: avaliar a Dependência da Prática de Karatê (DPK) em atletas e desportistas para determinar a prevalência e o grau de dependência e sua associação a comportamentos de risco. Métodos: foram recrutados 46 indivíduos de ambos os sexos, com média de $45,4(10,6)$ anos, $74,8(10,7) \mathrm{kg}, 173,1(7,2) \mathrm{cm}$, cujo índice de massa corporal de $24,89(2,93) \mathrm{kg} / \mathrm{m}^{2}$ os classificou como eutróficos. Nós consideramos medidas antropométricas, índices de uma escala de dependência adaptada para DPK, e informações sobre a percepção da autoimagem corporal. Resultados: foi constatado um índice significativamente maior de DPK em atletas mais experientes, porém quanto à insatisfação da autoimagem corporal, esta foi significativa apenas no grupo de praticantes menos experientes. Conclusão: a aplicação da Escala de DPK mostrou-se útil na detecção de dependência em praticantes de karatê em relação ao tempo de treino, embora não se tenha indicação de desdobramentos negativos advindos deste quadro de dependência. Recomenda-se a aplicação desta escala para as diferentes artes marciais, a fim de determinar, nos diferentes estilos, as motivações e possíveis consequências de um engajamento patológico no esporte.

Palavras-chave: artes marciais, exercício, questionário.

\section{ABSTRACT}

Introduction: martial arts training has been showing tremendous growth at both entertainment and competitive levels. Although the benefits of regular exercising are undeniable, we have growing evidence of excessive practice among athletes and sportsmen, with presence of pathological addiction traits. Physical Exercise Addiction (PEA) is displayed in primary and secondary forms. Objective: our goal is to assess the Karate Practice Addiction (KPA) in athletes and sportsmen determining its prevalence and dependence levels, as well as associations with risky behaviors. Methods: we studied 46 individuals, from both genders, with mean age $45.4(10.6), 74.8(10.7) \mathrm{kg}, 173.1(7.2) \mathrm{cm}$, for which the body mass index $24.89(2.93) \mathrm{kg} / \mathrm{m}^{2}$ established an eutrophic pattern. We gathered anthropometric measurements, scores from an addiction scale adapted for KPA, and body image perception data. Results: seasoned athletes showed a significantly higher KPA score, while less experienced practitioners were significantly less satisfied with their body image. Conclusion: the KPA scale proved useful to correlate addiction and time of engagement to Karate. We recommend the use of this scale as an assessment tool to determine motivations and risks of pathological behavior in Karate and other martial arts.

Keywords: martial arts, exercise, questionnaires.

\section{RESUMEN}

Introducción: las artes marciales se han transformado en actividades con gran crecimiento, ya sea desde el punto de vista recreacional o competitivo. Aunque sean innegables los beneficios de la práctica regular de ejercicios físicos, aumentan las evidencias de que el exceso de ejercicio entre atletas y deportistas de diferentes modalidades deportivas puede llevar a la dependencia e incluso a situaciones patológicas. Este exceso denominado Dependencia de la Práctica del Ejercicio (DPE) puede presentarse en la forma primaria o secundaria. Objetivo: evaluar la Dependencia de la Práctica de Karate (DPK) en atletas y deportistas para determinar la prevalencia y el grado de dependencia y asociar a potenciales consecuencias negativas o riesgos. Métodos: fueron reclutados 46 individuos de ambos sexos con promedio de edad de $45,4(10,6)$ años, $74,8(10,7) \mathrm{kg}, 173,1(7,2) \mathrm{cm}$ cuyo índice de masa corporal de $24,89(2,93) \mathrm{kg} / \mathrm{cm}^{2}$ los clasificó como eutróficos. Además de la evaluación antropométrica fue aplicada la Escala de Dependencia de Práctica de Karate (DPK) adaptada para las artes marciales y una escala de evaluación para la autoimagen corporal. Resultados: fue constatado un aumento significativo de DPK en atletas con más experiencia, pero cuanto a la insatisfacción de la auto imagen corporal, ésta fue significativa apenas en el grupo de practicantes con menor experiencia. Conclusión: la aplicación de la Escala de DPK se 
mostró útil en la detección de la dependencia en practicantes de karate en relación al tiempo de práctica, aunque no hubo manifestación negativa como consecuencia de este cuadro de dependencia. Se recomienda la aplicación de esta escala para las diferentes artes marciales a fin de determinar en los diferentes estilos las motivaciones y posibles consecuencias de algún problema patológico en el deporte.

Palabras clave: artes marciales, ejercicio, cuestionarios.

\section{INTRODUÇÃO}

As artes marciais têm se tornado as atividades mais praticadas e populares no mundo, tanto no âmbito recreacional, como competitivo, com cerca de 6,9 milhões de participantes só nos EUA, segundo a Martial Arts Industry Association (MAIA) ${ }^{1,2}$. Embora existam diferentes estilos de artes marciais e esportes de combate originários do Japão, Okinawa, Korea, China, Filipinas, Brasil, França, Israel e América há diversas similaridades em relação às técnicas e métodos de treinamento, sendo que algumas destas datam de milhares de anos, conforme referenciado em relatos na Grécia antiga e Egito'. As artes marciais, incluindo o karatê, são formas milenares de combate, modificadas para o esporte moderno, e está bem estabelecido o seu poder de promoção para a saúde, bem estar físico e psicológico ${ }^{2}$. O Karatê, ou caminho das mãos vazias, é uma das versões japonesas para a arte do combate desarmado; sendo Gichin Funakoshi (1957 - 1969) o fundador do principal estilo moderno: Shotokan. O treino tradicional consiste na prática de três habilidades: Kihon, Kata e Kumitê. O Kihon consiste no treino de técnicas básicas como socos, chutes, bloqueios e ataques que são executados ou em posição estática ou em movimento em diversas posturas (bases). Os Katas são séries de formas em uma sequência pré-estabelecida de técnicas de defesa e ataque em movimento. O Kumite ou luta, é a execução de técnicas de defesa e ataque em movimentação livre contra um oponente ${ }^{3,4}$.

Embora seja inegável, por décadas de pesquisas epidemiológicas, que estabelecem consenso entre prática regular de atividade física e inumeráveis benefícios físicos e psicológicos, nas últimas décadas há a preocupação com o excesso da prática de exercícios, tanto em desportistas como atletas ${ }^{5,6}$. Este excesso, denominado de síndrome da dependência da prática de exercícios físicos (DEF) foi inicialmente descrita na década de 70 por Baekeland, em sua pesquisa com corredores regulares, que treinavam 5 vezes por semana e a recusa em interromper a prática esportiva, mesmo com incentivo financeiro; e sintomas de abstinência nos praticantes moderados que aderiram ao estudo ${ }^{7}$.

O excesso da prática de exercícios, muitas vezes incentivado pelo marketing das indústrias de fitness, pode desencadear comportamentos compulsivos chegando mesmo ao estresse físico ${ }^{6,8}$. Desde 1970 já existem relatos de síndrome de abstinência relacionados à prática excessiva de exercícios, implicando inicialmente em alterações no padrão de sono ${ }^{7}$; desde então tal padrão comportamental já foi designado desde positive addiction (dependência positiva) ${ }^{8}$ até a negative addiction (dependência negativa) $)^{9,10}$.

No âmbito da pesquisa no Brasil já foi validado um questionário que avalia DEF em maratonistas ${ }^{8}$ e suas repercussões negativas, tradicionalmente descritas na população de atletas principalmente em maratonistas ${ }^{8,11}$; porém alguns outros trabalhos já foram realizados com fisiculturistas e levantadores de peso ${ }^{12}$; praticantes de corrida de aventura6; ultra maratonistas ${ }^{13}$ e até mesmo em atletas de karatê estilo Shotokan ${ }^{14}$. Em relação a este último público já foi registrado que há tendência dos atletas de karatê Shotokan exibirem um perfil de comprometimento à prática relacionado ao estilo de vida, mais do que uma dependência propriamente dita, abrindo a perspectiva de que a DEF esteja relacionada ao tipo de exercício praticado, além de outras variáveis com tempo de atividade, intensidade, frequência etc ${ }^{10,14,15}$.

Uma consequência negativa da DPEF bastante estudada em atletas e desportistas, é a sua relação com distorção de imagem corporal e transtornos alimentares ${ }^{16}$. A imagem corporal (IC) é definida como aquela que temos em nossas mentes do tamanho, contorno e da forma de nossos corpos, bem como nos sentimentos relacionados a suas características e às suas partes constituintes ${ }^{17}$. A IC é constituída por dois componentes: 0 perceptivo (percepção corporal) e o atitudinal (sentimentos, pensamentos e ações sobre o corpo), e a insatisfação pode ser descrita como a discrepância entre o corpo ideal e percebido ${ }^{18}$. Em relação a autoconcepção que temos de nosso corpo é documentado que muitas vezes o início da prática de atividade física é motivado pela insatisfação, tanto do próprio corpo como da imagem corporal. Esta insatisfação corporal e a busca por um corpo "ideal" pode levar à adoção de atitudes inadequadas em relação ao exercício físico, possibilitando o desenvolvimento de uma dependência secundária, caracterizada por um controle patológico do peso e/ou composição corporal, com possibilidade de associação com transtornos alimentares como a anorexia e a bulimia nervosa ${ }^{16}$.

O objetivo do trabalho foi avaliar o grau de dependência da prática exercícios físicos em amostra de atletas de alto nível de karatê estilo Shotokan em comparação a praticantes aderentes, a fim de determinar além da prevalência de indivíduos dependentes, a sua relação com possíveis efeitos negativos e potenciais riscos associados.

\section{CASUÍSTICA}

Participaram do estudo 46 indivíduos, sendo 23 atletas faixas pretas do 1 ao 4․ grau ou dan (17 homens e 5 mulheres) e 23 praticantes faixas coloridas (19 homens e 4 mulheres), foram recrutados em um dojô com mais de 45 anos de tradição no ensino de Karatê estilo Shotokan na cidade de São Paulo SP, Brasil. Como critérios de seleção foram observados serem maiores de 21 anos, ambos os sexos, com prática regular de karatê, frequência de no mínimo $2 x /$ semana, tanto com finalidade recreacional como competitiva. Dentre a amostra de faixas pretas estavam inclusos indivíduos com ampla experiência em campeonatos nacionais e internacionais entre estes campeões brasileiros, universitários, sulamericanos, panamericanos e mundiais, todos assinaram um termo de consentimento livre e esclarecido conforme modelo aprovado por um Comitê de Ética (COEP122/010). Após foram coletadas informações sobre identificação, data nascimento, tempo total de treino de karatê e se faixa preta, o tempo total de anos de graduação.

Para a medida da massa corporal foi empregada balança portátil digital modelo TBF 551 (Tanita ${ }^{\circledR}$, Japão), com precisão de 0,1 kg e capacidade total de $136 \mathrm{~kg}$. A estatura foi obtida através de antropômetro portátil Body meter 208 (Seca ${ }^{\circledR}$, United Kingdom), total $200 \mathrm{~cm}$, precisão de 1 $\mathrm{cm}$. Através da relação entre massa corporal e estatura ao quadrado foi calculado o Índice de Massa Corporal (IMC: $\mathrm{kg} \bullet \mathrm{m}^{-2}$ ); e o estado nutricional classificado segundo OMS (2004).

Para avaliar o grau de Dependência da Prática de Karatê (DPK) foi aplicado Questionário"Negative Addiction Scale"mo inicialmente construído para avaliação da dependência em corredores ${ }^{15}$ e validado para a população brasileira ${ }^{8}$. O mesmo foi modificado para praticantes de karatê Shotokan onde a palavra "corrida" do questionário original, foi alterada para "treino de karatê"; tal modificação já foi empregada em outras pesquisas, o que possibilita determinar a DEF em todos tipos de exercício ${ }^{19}$. O questionário 
de Dependência da Prática de Karatê (DPK) consistiu de 13 perguntas, totalizando 14 pontos. A pontuação atribuída seguiu aos seguintes critérios: 1 pontos para as questões 1(a); 2(a) ou (b); 3(b); 4 até 9,11,12="discordo muito"; 10="concordo muito"e 13(b) ou (h). O somatório dos pontos atingidos por cada indivíduo permitiu classificá-lo em três categorias: a) baixa dependência (0 - 4 pontos); moderada dependência (5 - 9 pontos) e alta dependência (10 - 14 pontos) $)^{8,10}$.

Para a avaliação da IC foi empregada uma escala de 10 silhuetas de ambos os sexos, por Harris e cols. ${ }^{20}$. Esta escala foi desenvolvida com base em fotografias trabalhadas no computador empregando o software Photoshop ${ }^{\circledR}$ (Adobe Systems; EUA) ${ }^{20,21}$. A escala correspondente ao sexo foi apresentada e questionada qual representaria respectivamente sua imagem atual e ideal. Várias revisões têm sugerido efetividade na determinação individual e do tipo corporal idealizado, sendo a discrepância entres estas duas medidas um forte indicativo de nível de insatisfação

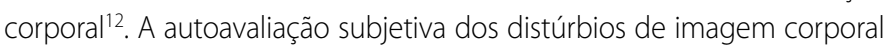
é em geral, estimada pelo emprego de Escalas de Silhuetas compostas por figuras especialmente construídas para esta finalidade ${ }^{20}$.

As variáveis contínuas foram analisadas descritivamente pelos valores de tendência central (média) e dispersão (desvio padrão). Para detectar diferenças estatísticas entre imagem atual e ideal entre faixas pretas e menos graduados, foi aplicado teste não paramétrico de Wilcoxon com um nível de significância de 5\% para rejeitar a Hipótese Nula $\left(\mathrm{H}_{0}\right)$. Para testar a hipótese de independência entre graduação e DPK esta foi realizada a partir da construção de uma tabela contingência. Um teste exato de Fisher aplicado a esta tabela que rejeitou $(p=0,04593)$ a hipótese de independência. Foi empregado para a realização das análises o programa estatístico R versão 2.10.1 da The R Foundation for Statistical Computing ${ }^{\circledR}$.

\section{RESULTADOS}

Os dados referentes ao total da amostra e categorias de faixa preta e colorida estão na tabela 1.

A amostra de faixas pretas apresentou em relação aos faixas coloridas, diferenças estatísticas $(p<0,01)$ em relação à média de idade e tempo de prática de karatê, não havendo diferença estatisticamente significativa para as variáveis de massa corporal, estatura e DPK. O IMC não apresentou diferença entre os grupos, classificando o estado nutricional de faixas pretas e coloridas como eutrófico ${ }^{22}$.

Em relação ao tempo total de obtenção do $1^{\circ}$ grau da faixa preta, este foi em média de 16,2 (8,3) anos variando entre 3 a 32 anos. Quanto a imagem corporal, houve diferença estatisticamente significativa $(p<0,05)$ para imagem atual e ideal apenas no grupo dos faixas coloridas.

Tabela 1. Perfil antropométrico e da prática de Karatê estilo Shotokan da amostra geral e por graduação. São Paulo, 2014.

\begin{tabular}{|c|c|c|c|c|c|c|c|c|c|}
\hline \multirow[b]{2}{*}{ Variáveis } & \multicolumn{3}{|c|}{ Geral $(n=46)$} & \multicolumn{3}{|c|}{ Faixas Pretas $(n=23)$} & \multicolumn{3}{|c|}{$\begin{array}{c}\text { Faixas Coloridas } \\
(n=23)\end{array}$} \\
\hline & \begin{tabular}{|l|} 
MD \\
(DP) \\
\end{tabular} & \begin{tabular}{|c|} 
Míni- \\
mo
\end{tabular} & $\begin{array}{c}\text { Máxi- } \\
\text { mo }\end{array}$ & \begin{tabular}{|l|} 
MD \\
(DP) \\
\end{tabular} & $\begin{array}{c}\text { Míni- } \\
\text { mo }\end{array}$ & \begin{tabular}{|c} 
Máxi- \\
mo
\end{tabular} & \begin{tabular}{|l|} 
MD \\
(DP) \\
\end{tabular} & $\begin{array}{c}\text { Míni- } \\
\text { mo }\end{array}$ & $\begin{array}{c}\text { Máxi- } \\
\text { mo }\end{array}$ \\
\hline Idade (anos) & $\begin{array}{l}45,4 \\
(10,6) \\
\end{array}$ & 21,0 & 68,0 & $\begin{array}{l}49,8 \\
(9,2)^{*}\end{array}$ & 32,0 & 68,0 & $\begin{array}{l}41,0 \\
(10,3)\end{array}$ & 21,0 & 63,0 \\
\hline $\begin{array}{c}\text { Massa corporal } \\
(\mathrm{kg})\end{array}$ & \begin{tabular}{|l|}
74,8 \\
$(10,7)$
\end{tabular} & 49,0 & 100,0 & \begin{tabular}{|l|}
74.2 \\
$(10.7)$
\end{tabular} & 51.0 & 91,0 & \begin{tabular}{|l|}
75,3 \\
$(11.0)$
\end{tabular} & 49,0 & 100,0 \\
\hline Estatura $(\mathrm{cm})$ & \begin{tabular}{|l|}
173,1 \\
$(7,2)$ \\
\end{tabular} & 157,0 & 187.0 & $\begin{array}{c}173,0 \\
(6,8) \\
\end{array}$ & 160,0 & 184,0 & \begin{tabular}{|c|}
173,3 \\
$(7,7)$ \\
\end{tabular} & 157,0 & 187,0 \\
\hline IMC $\left(\mathrm{kg} \cdot \mathrm{m}^{-2}\right)$ & $\begin{array}{l}24,89 \\
(2,93) \\
\end{array}$ & 19,88 & 34,25 & $\begin{array}{l}24,72 \\
(2,55) \\
\end{array}$ & 19,92 & 29,40 & $\begin{array}{l}25,06 \\
(3,31) \\
\end{array}$ & 19,88 & 34,25 \\
\hline Prática (anos) & \begin{tabular}{|l|}
17,9 \\
$(12.3)$ \\
\end{tabular} & 1,0 & 40.0 & $\begin{array}{l}25,9 \\
(9,2)^{*}\end{array}$ & 8,0 & 40,0 & $\begin{array}{c}9,8 \\
(9,4) \\
\end{array}$ & 1,0 & 30,0 \\
\hline Imagem atual & $\begin{array}{c}5,3 \\
(1,5) \\
\end{array}$ & 2,0 & 9,0 & $\begin{array}{c}5,4 \\
(1,6)\end{array}$ & 2,0 & 8,0 & \begin{tabular}{|c|}
5,3 \\
$(1,4)$ \\
\end{tabular} & 3,0 & 9,0 \\
\hline Imagem ideal & $\begin{array}{c}5,0 \\
(1,5) \\
\end{array}$ & 2,0 & 9,0 & $\begin{array}{c}5,3 \\
(1,7)\end{array}$ & 2,0 & 8,0 & $\begin{array}{c}4,8 \\
(1,5) \#\end{array}$ & 2,0 & 8,0 \\
\hline Escore DPK & $\begin{array}{l}6,3 \\
(2,2) \\
\end{array}$ & 2,0 & 10,0 & $\begin{array}{c}6,7 \\
(2,3)\end{array}$ & 2,0 & 10,0 & $\begin{array}{c}6,0 \\
(2,0)\end{array}$ & 3,0 & 10,0 \\
\hline
\end{tabular}

Legenda: MD: Média, DP: Desvio Padrão, IMC: Índice de Massa Corporal; DPK: Dependência da Prática de Karatê. *diferença estatística entre grupos. \#diferença estatística intra grupos
Na tabela 2, estão os resultados da escala de DPK, e de acordo com a análise estatística, há relação entre dependência e graduação. O grupo dos faixas pretas apresentou maior número de indivíduos classificados entre dependência moderada-alta e os coloridas baixa-moderada.

Tabela 2. Número de caratecas classificados conforme níveis de Dependência da Prática de Karatê (DPK) entre as categorias de faixas pretas e coloridas.

\begin{tabular}{c|c|c|c|c}
\hline & $\begin{array}{c}\text { Baixa } \\
\text { dependência }\end{array}$ & $\begin{array}{c}\text { Média } \\
\text { dependência }\end{array}$ & $\begin{array}{c}\text { Alta } \\
\text { dependência }\end{array}$ & Total \\
\hline Faixas Pretas & 1 & 16 & 6 & 23 \\
\hline Faixas Coloridas & 5 & 17 & 1 & 23 \\
\hline Total & 6 & 33 & 7 & 46 \\
\hline
\end{tabular}

\section{DISCUSSÃO}

Na presente investigação pretendeu-se estudar a Dependência da Prática de Karatê (DPK) em uma amostra de atletas experientes, em contraposição a praticantes menos graduados e os possíveis impactos negativos relacionados aos graus de dependência. O objeto desta questão se baseia em estudos prévios com praticantes de artes marciais e relatos de distúrbios severos de humor em decorrência da privação do treino, além de resultados conflitantes, quando da abstinência da prática de karatê, variando desde "distúrbios emocionais em níveis dolorosos"14 a"mudanças significativas em sintomas físicos"23. Outro fator de investigação reside no motivador pela prática do exercício e da relação com alterações psicológicas da privação, sendo que estas foram mais pronunciadas para aqueles indivíduos que referiam engajamento na prática por motivos de saúde do que por outras razões ${ }^{14,24}$.

Segundo Obodyński et $a l^{25}$ na investigação sobre a motivação para a prática de artes marciais e sua hierarquia na influência da formação da personalidade, observaram que há uma escala de valores (por exemplo: segurança própria e familiar, felicidade, prazer, paz etc) que diferencia a motivação para a prática de uma arte marcial, portanto é de se esperar que qualquer interrupção na rotina de treinamento resulte em maiores distúrbios de natureza psicológica do que física ${ }^{14}$. Outra questão que pode estar relacionada, diz respeito ao volume de treinamento e/ou intensidade, onde foram constatadas alterações negativas no humor em praticantes habituados, apenas quando da redução da intensidade do treinamento, e não de ambas variáveis, indicando que pode haver compensação da perda de volume de treinamento pela intensidade ${ }^{26}$.

Existe ainda uma lacuna nos estudos na área de DPE, em identificar o grau de comprometimento do individuo com o exercício, que se apresenta desde um engajamento saudável até uma dependência patológica ${ }^{15}$. Neste aspecto empregamos um questionário validado para população brasileira para o estudo da DEF em corredores $^{8}$, que permite classificar o indivíduo em três categoria de dependência: baixa, moderada e alta. Para sua aplicação ocorreu a troca, em relação ao questionário original da palavra "corrida" para "treino de karatê" conforme realizado em diversos estudos com diferentes modalidades esportivas $5^{6,19}$. Szabo e Parkins ${ }^{14}$ empregaram a mesma técnica e confeccionaram a escala denominada Commitment to Martial Art (CMA), em atletas de karatê Shotokan, sem afetar as propriedades psicométricas da escala de avaliação original ${ }^{14}$. Nossa escolha em empregar o questionário de DPK, ao invés do CMA residiu no fato de buscar um refinamento na discussão sobre o grau de dependência, permitindo não só a detecção dos indivíduos dependentes, mas diferentes nuance de relação entre o grau de dependência (baixa, moderada e alta), com o nível de treinamento (faixa preta versus coloridas). Tal abordagem já foi explorada em um trabalho entre atletas profissionais e amadores de modalidades coletivas (vôlei, futebol, futsal, handebol, basquete) ou individuais (atletismo, natação, fisiculturismo, ginástica artística), cujo objetivo foi verificar diferenças nos escores de DEF e qualidade de vida ${ }^{19}$. Como resultados principais, os autores constataram que percentualmente atletas profissionais (33,3\%) e amadores (36,5\%) possuem escores similares de DEF, porém há maiores escores em atletas amadores de modalidades coletivas 
(40,0\%) do que individuais (32,5\%); já quando da análise entre atletas profissionais há inversão nos resultados, com escores de DEF maiores entre as modalidades individuais (43,7\%) do que coletivas (25,0\%). Estes resultados estão de acordo com que foi constatado em nosso estudo, onde o grau de profissionalismo (faixas pretas) se relacionou com maiores escores de DPK, com a diferença de investigarmos graus de dependência e experiência em uma modalidade individual especifica: o Karatê Shotokan.

Apesar desta investigação não compreender o estudo da abstinência à prática de Karatê, nossos resultados permitem especular, baseado no trabalho de Szabo e Parkin ${ }^{14}$ em praticantes de karatê estilo Shotokan e sintomas de privação do treino por uma semana, que os caratecas mais experientes e com maior DPK em ambos os sexos podem experimentar sintomas de abstinência da prática. Tais constatações indicam, conforme discutido anteriormente, que o questionário de DPK possui amplo espectro de aplicação e detecção de dependência, podendo servir de instrumento para avaliação e acompanhamento de rotina de treinamento em atletas de diferentes modalidades, inclusive nas artes marciais, principalmente nos períodos de pausa para recuperação por estresse pela própria rotina de treino ou por lesões inerentes à própria modalidade esportiva, onde possivelmente o grau de dependência possa se manifestar em sintomas psicológicos e físicos de abstinência ${ }^{14,15}$

Uma das repercussões negativas da DEF tanto em praticantes como atletas é a insatisfação com imagem corporal podendo desencadear uma dependência secundária ao exercício. Como diagnóstico hierárquico entre DEF Primária, teríamos que neste caso o exercício seria a própria motivação, não excedendo uma preocupação aparentemente saudável com a prática de exercícios físicos ${ }^{27}$; já na DEF Secundária a principal motivação seria o controle e manipulação da composição corporal ${ }^{11}$. Embora não seja uma regra, sintomas de dependência secundária são observados em indivíduos com transtornos alimentares: anorexia nervosa (AN) e bulimia (BN), e estima-se que cerca de $80 \%$ dos pacientes com AN e 55\% com BN pratiquem atividade física excessiva e inadequada no percurso de sua história clínica ${ }^{16}$.

Foram observadas alterações na percepção da imagem corporal apenas para o grupo de caratecas não graduado. Neste grupo, a diferença entre imagem atual e desejável, embora significativa, se apresentou com uma pequena variação, respectivamente de 5,3 $(1,4)$ para 4,8 $(1,5)$. Já no grupo com maior graduação, tempo prática e escore de DPK não foram observadas diferenças significativas da imagem corporal. Estes resultados indicam globalmente que a prática de Karatê não se apresenta como um gatilho ou mesmo um reforço de desenvolvimento de atitudes negativas tanto de percepção/atitude como em relação ao corpo e suas partes constituintes, sugerindo o contrário, que a prática traduz em melhores perspectivas de gerenciamento do componente de auto conhecimento.

\section{REFERÊNCIAS}

\section{Terry CM. The martial arts. Phys Med Rehabil Clin N Am 2006;17(3):645-676.}

2. Woodward TW. A Review of the effects of martial arts practice on health. WMJ. 2009;108(1):40-3.

3. Rossi L, Silva RC, Tirapegui J. Avaliaçăo nutricional de atletas de karatê. Rev APEF. 1999;14(1):40-9.

4. Rossi L, Tirapegui J. Avaliaçăo antropométrica de atletas de Karatê. R Bras Ci e Mov. 2007;15(3):39-46.

5. Lejoyeux M, Avril M, Richoux C, Embouazza H, Nivoli F. Prevalence of exercise dependence and other behavioral addictions among clients of a Parisian fitness room. Compr Psychiatry. 2008;49(4):353-8.

6. Antunes HK, Andersen ML, Tufik S, Mello MT. O estresse físico e a dependência de exercício físico. Rev Bras Med Esporte. 2006;12(5): 234-8.

7. Baekeland F. Exercise deprivation. Sleep and psychological reactions. Arc Gen Psychiatry. 1970;22(4):365-9.

8. Rosa DA, Mello MT, Souza-Formigoni MLO. Dependência da prática de exercícios físicos: estudos com maratonistas brasileiros. Rev Bras Med Esporte. 2003;9(1):9-14

9. Furst DM, Germone K. Negative addiction in male and female runners and exercises. Percept Mot Skills. 1993;77(1):192-4

10. Hailey BJ, Bailey LA. Negative addiction in runners: a quantative approach. J Sport Behav. 1982;5(2):150-4.

11. de Coverley Veale DM. Exercise dependence. Brit J Addict.1987;82:735-40.

12. Hurst R, Hale B, Smith D, Collins D. Exercise dependence, social physique anxiety, and social support in experienced and inexperienced bodybuilders and weightlifters. Br J Sports Med. 2000;34(6):431-5.

13. Allegre B, Therme P, Griffiths M. Individual factors and the context of physical activity in exercise dependence: a prospective study of 'ultra-marathoners'. Int J Ment Health Addiction. 2007;5(3):233-43.

14. Szabo A, Parkin AM. The psychological impact of training deprivation in martial arts. Pshycol Sports Exerc 2001;2(3):187-99

15. Hausenblas HA, Downs DS. Exercise dependence: a systematic review. PSYSPO. 2002;3(2):89-123.

16. Teixeira $P C$, Hearst N, Matsudo SM, Cordás TA, Conti MA. Adaptação transcultural: tradução e validação de conteúdo da versão brasileira do Commitment Exercise Scale. Rev Psiq Clin. 2011;38(1):24-8.
Kravchychyn et al. ${ }^{28}$ também observaram em seu estudo com atletas feminina de diferentes modalidades em equipe (basquetebol, handebol, vôlei e futsal), que apesar desta apresentarem distorção da autoimagem corporal, não tiveram propensão ao desenvolvimento de transtornos alimentares. Já Swami et al..$^{29}$ avaliando a insatisfação corporal entre praticantes do sexo feminino de corrida e artes marciais (taekwondo) observaram que no grupo das corredoras, onde o ideal corporal do esporte enfatiza menores percentuais de gordura e magreza, foi registrado maior grau de insatisfação do que nas lutadoras de artes marciais, onde o componente estético não é tão enfatizado, embora seja um esporte que tenha controle de peso. Reforçando nossos resultados Szabo e Parkin ${ }^{14}$ não puderam determinar para a mesma população de nosso estudo, atletas de karatê Shotokan ambos os sexos, se os sintomas de interrupção por uma semana nos treinamentos foram devidos a abstinência, por uma resposta à DPK caracterizando uma dependência secundária; ou apenas um reflexo da alteração do estilo de vida pela ausência do componente de exercício nas atividades de vida diárias, caracterizando uma dependência primária. Outro dado que reforça o componente primário da DPK foi a ausência de diferença entre os escores para o sexo masculino e feminino, resultado contrário à maioria dos trabalhos sobre DEF entre os gêneros, que indicam que as mulheres apresentam maiores amplitudes de sintomas de dependência quando comparados aos homens ${ }^{30}$.

\section{CONCLUSÃO}

A Escala de Dependência da Prática de Exercícios Físicos é um instrumento com amplo espectro de aplicações na detecção do grau de dependência, tanto em atletas amadores como profissionais, assim como instrumento de acompanhamento e gerenciamento de rotinas de interrupção de treinamento, para previsão de possíveis sintomas de abstinência com repercussões físicas e/ou emocionais. Os atletas de Karatê exibiriam graus diferentes de dependência que foram mais pronunciados nos mais experientes e graduados do que nos menos experientes. Apesar desta diferença significativa esta parece se caracterizar pela DPK primária, sem que haja evidência de uma evolução negativa, tanto em relação a percepção da imagem corporal como possível desenvolvimento de dependência secundária ao longo dos anos de engajamento, prática e aperfeiçoamento na arte marcial. Recomenda-se a aplicação da Escala de DPK em diferentes estilos de artes marciais para avaliar o impacto da dependência em cada modalidade, para determinar as nuances do engajamento entre desportista e atletas, assim como seu impacto e desdobramentos futuros.

Todos os autores declararam não haver qualquer potencial conflito de interesses referente a este artigo.

17. Slade PD. Body image in anorexia nervosa. Br J Psychiatry 1988;153(2):20-2.

18. Slade PD. What is body image? Behav Res Ther. 1994;32(5):497-502

19. Modolo VB, Mello MT, Gimenez PRB, Tufik S, Antunes HKM. Dependência de exercício físico: humor, qualidade de vida em atletas amadores e profissionais. Rev Bras Med Esporte. 2009;15(5):275-9.

20. Harris CV, Bradlyn AS, Coffman J, Gunel E, Cottrell L. BMI-based body size guides for women and men: development and validation of a novel pictorial method to assess weight-related concept. Inter J Obes. 2008;32(2):336-42.

21. Moraes C, Anjos LA, Marinho SMSA. Construção, adaptação e validação de escalas de silhuetas para autoavaliação do estado nutricional: uma revisão sistemática da literatura Cad Saúde Pública. 2012;28(1):7-19.

22. Organizaçăo Mundial de Saúde (OMS). Obesidade: prevenindo e controlando a epidemia global. São Paulo: Roca; 2004.

23. Gauvin L, Szabo A. Application of the experience sampling method to the study of the effects of exercise withdrawal on well-being. J Sports Exer Psychol. 1992;14(4):361-74.

24. Robbins J M, Joseph P. Experiencing exercise withdrawal: Possible consequences of therapeutic and mastery running. J Sport Psych. 1985;7(1):23-39.

25. Obodynsky K, Cynarski WJ, Withkowshi K. Report from research the perception of martial arts: case study of southern Poland. Ach Budo. 2008;4(1):78-81.

26. Witting AF, McConell GK, Costill DL, Schurr KT. Psychological effects during reduced training volume and intensity in distance runners. Int I Sports Med. 1992;13(6):497-9.

27. Bamber DJ, Cockerill IM, Rodgers S, Carroll D. Diagnostic criteria for exercise dependence in women. $\mathrm{Br}$ Sports Med. 2003;37(5):393-40.

28. Kravchychyn ACP, Silva DF, Machado FA. Relação entre estado nutricional, adiposidade corporal, percepção de autoimagem corporal e risco para transtornos alimentares em atletas de modalidades coletivas do gênero feminino. Rev Bras Educ Fís Esporte. 2013;27(3):459-66.

29. Swami V, Steadman L, Martin JT. A comparison of body size ideals, body dissatisfaction, and media influence between female track athletes, martial artists, and non-athletes. Psychol Sport Exerc. 2009;10(6):609-14

30. Davis C, Fox J. Excessive exercise and weight preoccupation in women. Addict Behav. 1993;18(2):201-11. 